\title{
e-Migrinter
}

$10 \mid 2013$

Genre et imbrication des rapports de domination dans les médias des minorités ethniques

\section{Des médias par et pour les minoritaires? (Re)production du genre et imbrication des rapports de domination}

Claire Cossée, Laura Navarro, Isabelle Rigoni et Eugénie Saitta

\section{(Q) OpenEdition}

Journals

Édition électronique

URL : https://journals.openedition.org/e-migrinter/461

DOI : 10.4000/e-migrinter.461

ISSN : 1961-9685

Éditeur

UMR 7301 - Migrinter

Édition imprimée

Date de publication : 11 avril 2013

Pagination : $3-13$

ISSN : 1961-9685

Référence électronique

Claire Cossée, Laura Navarro, Isabelle Rigoni et Eugénie Saitta, « Des médias par et pour les minoritaires? (Re)production du genre et imbrication des rapports de domination », e-Migrinter [En ligne], 10 | 2013, mis en ligne le, consulté le 20 mai 2021. URL : http://journals.openedition.org/emigrinter/461 ; DOI : https://doi.org/10.4000/e-migrinter.461 


\title{
Des médias par et pour les minoritaires ? (Re)production du genre et imbrication des rapports de domination
}

\author{
Claire Cossée, Laura Navarro, Isabelle Rigoni \& Eugénie Saitta
}

C

e numéro est issu du colloque international organisé en mars 2010 à la MSHS de Poitiers par l'équipe MINORITYMEDIA ${ }^{1}$ sur « les médias des minorités ethniques, entre hégémonie et résistances ? ». Il reprend particulièrement les textes de la session consacrée au genre et à l'articulation des rapports de domination. Notre réflexion est ancrée dans une tradition intellectuelle qui analyse les pratiques culturelles au cœur même des systèmes hégémoniques. Nous nous appuyons sur la notion d'hégémonie élaborée par Antonio Gramsci selon laquelle la famille, l'école, les médias ou les églises fonctionnent comme des instruments de socialisation et de propagation des valeurs dominantes, contribuant ainsi à l'élaboration d'un consensus plus durable que celui qui émane de la violence légitime (Altamirano, 2002).

Autrement dit, les appareils de l'hégémonie - c'est-à-dire, les institutions qui configurent l'économie, la culture, l'opinion publique et les mobilisations sociales - permettraient de diriger intellectuellement, moralement et politiquement une société, sans besoin

d'avoir recours à la violence physique pour obtenir le consensus de la majorité

(définition empruntée à Giner et al., 2004).

\footnotetext{
${ }^{1}$ Minoritymedia est une équipe d'excellence Marie Curie (FP6, 2006-2010, Université de Poitiers MIGRINTER) dont les recherches ont porté sur les médias créés par et/ou pour des minorités ethniques dans huit pays (Allemagne, Espagne, France, GrandeBretagne, Hongrie, Italie, Pays-Bas, Turquie).
}

Ainsi, nous nous intéressons à la façon dont des groupes sociaux intériorisent ou non et, à l'inverse, se démarquent ou non, des pratiques et discours hégémoniques, (re)produits en particulier par les médias de masse $^{2}$. Plusieurs aspects retiennent particulièrement notre attention. D'une part, du fait de l'ampleur de la diffusion, les jeux de pouvoir qui sous-tendent la relation entre médias et publics ont des conséquences sociales et culturelles importantes dans la mesure où ils renvoient à des enjeux majeurs en matière de propagande, de publicité ou d'éducation. D'autre part, les médias de masse tendent à imposer un modèle journalistique uniformisé, en particulier en termes de recrutement, de rapport hiérarchique, de répartition genrée des tâches, de rubricage, de choix des sources. Nous nous interrogeons sur les défis apportés, ou non, par les médias des minorités ethniques en la matière, principalement au regard des rapports de genre. Quelles sont leurs stratégies de contournement et de résistance, et jusqu'à quel point celles-ci fonctionnent-elles? Les producteurs des "médias des minorités ethniques $»^{3}$ revendiquent souvent leur positionnement comme allant à l'encontre

\footnotetext{
${ }^{2}$ Les «médias de masse » se caractérisent par: une audience massive, l'unidirectionalité et la centralisation des messages homogénéisants, et dans le cas des médias audiovisuels, une programmation continue (Altamirano, 2002).

3Par "médias des minorités ethniques", nous entendons les médias créés majoritairement par et pour les personnes immigrées et leurs descendants, les minorités religieuses, les minorités linguistiques et historiques. Pour une réflexion sur ce choix terminologique, lire Rigoni, 2010c.
} 
de la représentation que font les médias de masse de l'immigration, des rapports sociaux dans les quartiers paupérisés et des pays du Sud. Dans le champ académique, des voix se sont prononcées en faveur de "nouvelles règles de convivialité qui procurent non seulement une base pour des principes égalitaires mais aussi les conditions pour une communication interculturelle et le développement d'un sens nouveau de la communauté » (Castles, Davidson, 2000, p. 8). Dans cette veine, les médias des minorités ethniques ont été présentés non seulement comme les vecteurs privilégiés de discours et de représentations alternatifs (Morley, 2000 ; Rodriguez, 2001; Cottle, 2000 ; Husband, 1994 ; Riggins, 1992), mais aussi comme des lieux où peuvent se construire et s'expérimenter de nouveaux modèles de citoyenneté (Isin, Wood, 1999 ; Bauböck, 1998 ; Joppke, 1998 ; Soysal, 1994), plus inclusifs (Coleman, Normann, 2000) et respectueux de la diversité sociale et culturelle. Dans le projet MiNORITYMEDIA, nous nous sommes également demandé si les pratiques journalistiques étaient «alternatives » par rapport à celles des médias de masse, notamment eu égard aux rapports genrés tels qu'ils s'incarnent dans les représentations produites par ces médias, mais aussi dans leurs pratiques de travail. Nous nous sommes ainsi intéressés aussi bien aux modalités de répartition des tâches qu'aux rapports de pouvoir et aux processus décisionnels au sein de ces médias.

Nos travaux ont montré qu'une partie des médias des minorités ethniques proposent une re-présentation (Cossée, 2010) $\mathrm{du}$ groupe différente, plus complexe que celle véhiculée au sein des médias hégémoniques. Mais aussi, qu'ils permettent une contribution accrue des minorités à l'espace médiatique et donc leur donnent potentiellement la parole au sein de l'espace public (Rigoni, 2010a ; Rigoni, Berthomière, Hily, 2010; Navarro, 2008). Il serait néanmoins naï de penser ces médias comme relevant tous de pensées et de pratiques alternatives (Rigoni, Saitta, 2012). Cet ensemble hétérogène se situe bien au cœur de la contradiction entre adhésion et résistance à l'égard des médias de masse et de leur vision du monde en ce qui concerne les questions relatives aux représentations de l'Autre et de l'ailleurs. En ce sens, les médias des minorités ethniques permettent d'interroger plus largement les rapports sociaux à l'œuvre entre majoritaires et minoritaires (Guillaumin, 1972 ; Juteau, 1999 ; Simon, 2006 ; Rabaud, 2012) ${ }^{4}$. Nous sommes parties du postulat selon lequel la production médiatique des minoritaires pourrait aussi contribuer à transformer les pratiques et représentations produites dans le champ médiatique. De même que des travaux féministes de l'espace francophone (Juteau, 1981) soulèvent l'importance épistémologique d'une recherche menée par les minoritaires. Perspective qui se développe aujourd'hui en France (Ait Ben Lmadani, Moujoud, 2008; Cossée, 2012b entre autres) - y compris via des traductions de travaux antérieurs (par exemple Oprea, 2012, 2004), à l'instar du corpus des Black feminists. C'est précisément le point de vue adopté par l'article de Carmen Diop dans ce numéro, et par les nombreuses références qu'elle mobilise.

Mais qu'en est-il d'une analyse genrée de ces médias et de leur rapport aux idéologies et pratiques hégémoniques: en termes de résistance aux représentations dominantes concernant les rapports de genre, mais aussi, en termes de possibilité d'expression des femmes, d'accession au travail journalistique, de possibilité de plaider leur cause?

\footnotetext{
${ }^{4}$ Nous nous situons dans la lignée des problématiques développées notamment par ces auteurs, à savoir que ce sont les rapports entre majoritaires et minoritaires qui nous intéressent. Les minorités sont avant tout des groupes placés dans une situation minoritaire, c'està-dire minorisés (Cossée, 2012a).
} 


\section{Genre, ethnicité, médias : un état des lieux}

Dans la littérature existante, peu de travaux abordent les médias et le journalisme dans une perspective qui articule genre et ethnicité, moins encore lorsqu'il s'agit des médias des minorités ethniques. Si des travaux ont permis l'introduction d'une perspective de genre dans la recherche sur le journalisme (Mattelart, 1986, 2003 ; Carter, Branston, Allan, 1998 ; Neveu, 2000 ; De Bruin, Ross, 2004; Chambers, Steiner, Fleming, 2004 ; Djerf-Pierre, 2005, 2007 ; Robinson, 2004; Byerly, Ross, 2006 ; Damian-Gaillard, Frisque, Saitta, 2010), la majeure partie d'entre eux traite des médias dominants et peu des médias des minorités ou des médias dits « alternatifs ».

Certains travaux se sont interrogés pour savoir si les médias, dans leur ensemble et leur diversité, servent «la cause des femmes ", à l'instar de la revue d'histoire Le Temps des médias (Blandin, Méadel, 2009). Dans ce numéro qui vise à faire le point sur la question, un article aborde le rôle des médias dans un combat de femmes issues de l'immigration visant à leur émancipation (Thiéblemont-Dollet, 2009). Cette recherche porte sur un mouvement controversé $(\mathrm{Ni}$ Putes Ni Soumises), tant il a pu produire une image ambiguë de certains groupes issus de l'immigration habitant des banlieues françaises. Ici, il s'agit plus d'analyser l'utilisation par ces militantes des médias dominants, à savoir les alliances nouées avec des journalistes de ces médias pour mettre leur combat sur l'agenda politique. L'auteure analyse tout particulièrement les stratégies mises en œuvre par ces militantes pour contrecarrer l'exclusion des femmes issues de l'immigration de la «représentation sociale et militante », dans le but de «se faire voir et entendre autrement ». D'autres recherches analysent les processus de production de l'information et les pratiques journalistiques du point de vue du genre et de l'ethnicité (Rigoni, 2011, 2010b).
Il demeure toutefois assez rare que des recherches soient consacrées tant aux représentations véhiculées qu'aux pratiques journalistiques et politiques. La plupart des travaux qui articulent genre et ethnicité en analysant les médias étudient les représentations à l'encontre des femmes immigrées dans les médias dominants (Ouali, Messian, 1999 ; Rodríguez García, 2005). Certains soulignent le peu d'intérêt porté par la presse (Calvo, 2001 ; Pérez, 2003 ; Masanet Ripoll, Ripoll Arcacia, 2008) ou la télévision (Bernárdez Rodal, 2007) aux conditions des femmes immigrées (les immigrés sont incarnés par le masculinneutre), ou encore la distorsion des images proposées par rapport à la réalité sociale vécue par les femmes immigrées. D'autres travaux analysent les représentations dominantes à l'encontre des femmes musulmanes et démontrent une absence généralisée de leur voix, ainsi qu'une vision culturaliste prédominante qui place l'islam comme le facteur explicatif par excellence de la discrimination de ces femmes et qui renforce à la fois les stéréotypes des femmes musulmanes comme essentiellement passives, victimes et voilées (Martín Muñoz, 2005). Un discours qui, comme l'ont souligné plusieurs auteurs, participe d'une des formes les plus importantes qu'adopte aujourd'hui l'islamophobie: celle qui s'articule à partir de la construction de la figure sociale de la "femme musulmane » qui semblerait avoir besoin d'être "sauvée » (Guénif Souilamas, 2007 ; Guénif, Macé, 2004) et qui, par ailleurs, n'est pas neuve en «Occident » (Navarro, 2010).

Dans le champ de la production scientifique anglo-saxonne, davantage de travaux articulent genre, ethnicité et médias, mais peu du point de vue des pratiques, à quelques exceptions près, à l'instar des travaux de Jinx Coleman Broussard sur les trajectoires de figures de femmes journalistes noires (Broussard, 2004). Aux États-Unis principalement, un champ de recherche se développe autour de genre, race et médias, surtout grand public (Biagi, Kern-Foxworth, 
1997). Quelques ouvrages collectifs font référence sur cette articulation en dépassant la seule analyse de contenu, en s'intéressant aussi à la question de la réception et de la production (Lind, 2004). Mais là encore, le point de vue des minoritaires est très peu abordé (Dente Roos, Cuillier, 2004).

Par ailleurs, même si un certain nombre de travaux analysent les médias des minorités ethniques comme vecteurs de « représentation », de mobilisations, ou moins souvent, de pratiques journalistiques, très peu introduisent, là encore, une perspective de genre.

\section{Analyser les mécanismes de représentation des femmes dans les médias des minorités ethniques}

Les médias des minorités ethniques, parfois situés à la croisée d'autres espaces sociaux (politique, universitaire, etc.), constituent-ils des lieux propices à la transformation des rapports sociaux inégalitaires tels qu'on les observe dans les modèles médiatiques dominants (par exemple, Löfgren-Nilsson, 2010)? Leurs liens avec les groupes ethniques minorisés les rendent-ils plus soucieux de l'égalité des sexes ? Par ailleurs, la survie financière de la plupart des médias des minorités ethniques est souvent un sujet de préoccupation majeur pour les bénévoles et les professionnels qui les animent. Différentes stratégies sont élaborées pour répondre aux contraintes organisationnelles qui caractérisent surtout les médias émanant du «Tiers Secteur de la communication $»^{5}$,

${ }^{5}$ Les traits caractéristiques principaux des médias du Tiers Secteur de la communication seraient les suivants : 1) médias privés sans but lucratif, 2) qui ont une finalité sociale, 3) qui sont gérés par des organisations sociales très diverses, 4) qui exercent le droit à l'information et à la liberté d'expression, 5) et qui sont ouverts à la participation la plus large possible en ce qui concerne la propriété du média et l'accès à l'émission (Définition extraite à partir des «Principes pour un cadre de régulation démocratique sur la radio et la télévision communautaire» (AMARC, 2008) et de la «Résolution du Parlement majoritaires dans certains pays comme les Pays-Bas et la France ${ }^{6}$. Quel est l'impact des conditions de production sur la place des femmes et les écarts sexués dans les médias des minorités ethniques? Observe-t-on la mise en place de structures et de processus décisionnels plus horizontaux? En matière de ressources humaines et de hiérarchies professionnelles, relève-t-on des logiques de distribution des femmes et des hommes selon les différents secteurs, spécialités, compétences journalistiques? Ou, au contraire, le manque de moyens des médias issus du Tiers Secteur d'une part, et les logiques mercantiles de ceux issus du secteur commercial d'autre part, favorisent-ils la reproduction des rapports de domination hommes-femmes, notamment des logiques d'inclusion et d'exclusion des postes de pouvoir dans les médias? Comment s'articulent les perceptions des compétences et incompétences des uns et des autres sur la base de stéréotypes genrés mais aussi ethniques? Enfin, il convient également de s'interroger sur l'impact des dimensions âge et ethnicité des journalistes et des responsables médiatiques sur le rôle et la participation des femmes dans l'espace journalistique.

Cette problématique générale peut se décliner en une série de questions concrètes. Dans quelle mesure les médias des minorités ethniques favorisent-ils l'accès des femmes au champ journalistique, aux activités médiatiques et, plus largement, à la parole publique ? Permettent-ils l'accès aux médias de femmes issues de catégories populaires et/ou faiblement dotées en capital culturel,

Européen du 25 septembre 2008, sur les médias associatifs en Europe » (2008/2011, INI).

${ }^{6}$ Le projet MiNORITYMEDIA a recensé 3613 médias des minorités ethniques (presse écrite, radio, télévision, sites web à contenu informationnel) dans 8 pays européens (Allemagne, Angleterre, Espagne, France, Hongrie, Italie, Pays-Bas, Turquie). Sur 3094 données exploitables relatives à leur répartition par secteur d'activité (public, commercial, associatif), la part de médias émanant du secteur associatif est majoritaire dans 5 des 8 pays de l'étude, celle-ci représentant notamment $79 \%$ aux Pays-Bas et $57 \%$ en France. 
économique et social? Ou, au contraire, favorisent-ils celles qui sont surdotées, produisant une accentuation des mécanismes de domination de classe parmi les minorités ethniques? Peut-on y observer des mécanismes de promotion des femmes à des fonctions de décision (Rigoni, 2010b) ? La tendance à la féminisation de la profession de journaliste décrite dans plusieurs sociographies relatives aux médias dominants en France (Marchetti, Ruellan, 2001 ; IFP, 2001 ; IFP, 1992) s'observe-t-elle dans les médias des minorités ethniques? L'accès des femmes à la profession de journaliste permet-elle d'enrayer les phénomènes de distribution genrée des thématiques, spécialités et compétences journalistiques? Plusieurs études ont montré que, dans les médias dominants, les femmes travaillent majoritairement dans des domaines informationnels qui sont considérés comme une extension de leurs responsabilités domestiques et du rôle qui leur est socialement assigné de soin et d'éducation (Neveu, 2000). Monica LöfgrenNilsson (2010) repère une typification de domaines de spécialité comme féminins ou masculins ("gender typing») dans la rédaction de la télévision publique suédoise $S V T$, les femmes étant par exemple tendanciellement affectées aux soft news et les hommes aux hard news. Qu'en est-il dans les médias des minorités ethniques?

La participation des femmes permetelle enfin de transformer leurs représentations dans ces médias, comme sujets et sources des produits médiatiques; de promouvoir d'autres façons de faire journalistiques (compétences, savoir-faire, etc.) ? Une littérature fournie nous renseigne sur les représentations stéréotypées des femmes dans les médias de masse et, par extension, dans la culture de masse. Nombre d'auteurs, proches des courants marxistes et des courants féministes, ont dénoncé une instrumentalisation des rapports sociaux de sexe à des fins marchandes, par une mise en scène stéréotypée des identités sexuelles (femme-objet) (Herne, 1993; Bosshart,
1991 ; Lavoisier, 1978). Les professionnels des médias se sont eux-mêmes penchés sur la question des représentations médiatiques, et plus particulièrement, des assignations et des statuts donnés aux paroles de femmes dans le traitement de l'information (Barré et al., 2000). Plus récemment, des rapports administratifs et parlementaires ont été publiés sur la question, dans la lignée des politiques d'égalité hommes-femmes, portées au niveau de l'Organisation des Nations-Unies et de l'Union européenne: une délégation sénatoriale, présidée par Gisèle Gautier, a effectué des auditions en 2006-2007 et rédigé un rapport sur le thème «Femmes et hommes dans les médias», et une commission de réflexion sur "l'image des femmes dans les médias ", présidée par Michèle Reiser et rapportée par Brigitte Grésy, a présenté un rapport de synthèse en septembre 2008 (Reiser, Grésy, 2008). Dans ces travaux, qui traduisent un intérêt politico-administratif pour le sujet, la principale préoccupation est celle de l'image des femmes dans la publicité, et plus généralement dans les médias, des stéréotypes qui y sont véhiculés, et des évolutions qui se font jour.

À l'inverse, peu de travaux concernent la représentation des femmes en général, et des femmes issues des minorités ethniques en particulier, que ce soit dans les médias adressés au grand public ou ceux adressés spécifiquement aux minorités ethniques. Nombres d'études historiques, sociologiques et d'analyses de discours ont souligné la représentation stéréotypée et la stigmatisation des immigrés et des minorités ethniques dans les médias de masse français (Bonnafous, 1991; Battegay, Boubeker 1993 ; Rigoni, 2007), espagnols (Bañón Hernández, 2002) et d'autres pays européens et d'Amérique latine (Frachon, Sassoon, 2008 ; van Dijk, 2003). Peu d'entre elles ont observé les effets de genre, à l'instar de Nacira Guénif Souilamas et Eric Macé (2004) qui ont montré comment les dominations ethniques et genrées opéraient sur la scène publique française, au détriment 
du «garçon arabe». Plus encore, la littérature demeure silencieuse sur les mécanismes de représentation des femmes dans les médias des minorités ethniques. Dans ce numéro, plusieurs articles apportent des éléments de compréhension de ces mécanismes et invitent à des recherches plus approfondies et systématiques.

\section{Articuler genre et ethnicité}

Ce numéro s'intéresse donc aux rapports de production entre femmes et hommes dans l'espace journalistique, ainsi qu'aux rapports sociaux de sexe présents dans les représentations produites par les médias des minorités ethniques. L'objectif est de comprendre comment le genre s'articule à l'ethnicité dans la division du travail (organisationnelle, statutaire, thématique) et dans les représentations produites.

Dans le premier article, Inaki Zabaleta, Nicolas Xamardo, Arantza Gutierez, Santi Urrutia et Itxaso Fernandez, proposent une analyse du profil professionnel des journalistes femmes travaillant dans les médias des minorités linguistiques en Europe. Ils s'intéressent plus précisément à deux formes potentielles d'inégalité genrée : la première concerne le recrutement des journalistes et la seconde leur niveau de salaire. Les résultats montrent que la part de femmes travaillant dans ces médias est particulièrement élevée et, surtout, qu'il n'existe pas de perception d'une inégalité de salaire entre hommes et femmes à compétences égales. Cependant, plusieurs journalistes de différentes minorités linguistiques reconnaissent que le plafond de verre ou encore les inégalités en matière d'avancement professionnel produisent une discrimination des femmes en termes de salaire.

Développant une analyse des rapports matériels de production dans l'espace journalistique des médias panafricains francophones, Carmen Diop propose un regard " de l'intérieur», de sa propre expérience journalistique, et clairement situé. Cet article offre une lecture des rapports de pouvoir au sein des rédactions à partir d'une approche intersectionnelle. Il s'inscrit également dans la sociologie des professions et du journalisme, tout en s'appuyant non seulement sur une analyse rétrospective d'une participation observante, mais aussi sur une enquête par entretiens. Ce texte avance l'hypothèse forte «que, sous couvert de s'adresser à un public africain avec des patrons et des rédacteurs africains, ces médias reproduisent le modèle, les rapports sociaux de sexe de la presse dominante et le déséquilibre Nord/Sud dans leurs modes de production dans un contexte néocolonial». Il s'inscrit dans un courant principalement mené et développé aujourd'hui par des minoritaires, et s'appuie sur divers corpus féministes afin de prolonger la réflexion épistémologique sur ce que les analyses des minoritaires permettent de révéler : comment et en quoi les chercheur(e)s situé(e)s du côté des pôles dominants tendent à proposer leurs travaux comme un savoir universel (et donc légitime), occultant par la même l'importance de leur position dans la production de ce savoir.

Les deux derniers articles de ce numéro analysent les représentations médiatiques des femmes immigrées dans des magazines féminins issus des minorités ethniques. Virginie Sassoon, à partir de son travail de thèse récemment soutenu, souligne les «ambiguïtés» de la presse féminine noire. Le travail a été conduit à partir d'une analyse des contenus, ainsi que sur une enquête auprès de lectrices et de productrices et producteurs des magazines Amina, Miss Ebène et Brune en France. Il est évident que ces médias cherchent également à proposer une représentation de soi alternative pour les femmes noires racisées et altérisées, souvent fortement érotisées dans la presse dominante. Mais ces magazines répondent aussi à l'invisibilisation de ces femmes, notamment dans la presse féminine blanche. S’inscrivant dans la lignée 
de la philosophie de la reconnaissance, l'auteure défend que "Les résultats de (sa) recherche montrent ainsi que ces magazines sont consommés dans le cadre de stratégies identitaires visant à compenser un sentiment d'invisibilité ainsi que la relative absence de figures "positives" dans la sphère médiatique dominante ». Positionnement ambigu certes, cependant l'auteure appelle de ses vœux le développement de la recherche sur les médias des minoritaires en France, tant ils nous apprennent sur les tensions vécues par les minoritaires eux-mêmes et peut-être encore plus ELLES-mêmes, au cœur des rapports de domination qui enferment et contre lesquelles ces médias se positionnent, parfois tout contre.

Pour sa part, Ana Mendieta s'interroge sur l'image des femmes dans les publications adressées spécifiquement à des femmes immigrées au Pays Basque espagnol. L'auteure expose d'abord le contexte, marqué par l'importante croissance des populations immigrées et l'éclosion des médias créés par et/ou pour celles-ci. Elle s'appuie ensuite sur des travaux consacrés aux représentations dominantes des femmes immigrées dans la presse espagnole pour les comparer à ses propres résultats portant sur quatre magazines basques adressés spécifiquement aux femmes immigrées: Mujeres del Mundo "Babel», Mujeres con Voq, Mujeres inmigrantes de San Ignacio et Euskadi News. À travers l'analyse de contenu de ces publications depuis 2007, elle constate que les femmes immigrées ont une visibilité plus importante comme protagonistes actives des articles qui les concernent - et non comme victimes de discrimination, employées de maison, mères ou épouses -, et sont l'objet d'un cadrage plus large de l'information qui permet de mettre en avant des sujets différents de ceux couverts habituellement par la presse espagnole généraliste, comme le travail des femmes immigrées qui sont des militantes ou des intellectuelles.
Les résultats de ces recherches permettent d'avancer dans la compréhension de l'articulation du genre et de l'ethnicité dans un espace journalistique particulier, tout en invitant à des recherches plus approfondies et systématiques dans un domaine qui reste très peu développé en Europe malgré les enjeux majeurs qu'il représente, dans des sociétés où les traces coloniales et patriarcales sont encore très présentes dans les relations économiques, politiques et culturelles.

\section{Claire Cossée}

Université Paris Est Créteil /

CIRCEFT-REV

CRESPPA-GTM

claire.cossee@gtm.cresppa.cnrs.fr

Laura Navarro

ERASME / Université Paris 8 launagar@yahoo.es

Isabelle Rigoni

MIGRINTER / Université de Poitiers isabelle.rigoni@club-internet.fr http://irigoni.blogspot.com/

Eugénie Saitta CRAPE / Université Rennes 1 eugenie.saitta@univ-rennes1.fr 


\section{Bibliographie}

Association Mondiale des Radiodiffuseurs Communautaires (AMARC) (2008) Principes pour un cadre de régulation démocratique sur la radio et la télévision communautaire, 3 mai.

Ait Ben Lmadani, Fatima ; Moujoud, Nasima (2008) À propos de la pensée majoritaire au sein du féminisme antiraciste universitaire en France, Journée d'etude "Classe des femmes et hétérogénéité du groupe des femmes: deux notions inconciliables? 》 (23 juin), Paris, RT $\mathrm{n}^{\circ} 24$ (AFS); GTM (Genre, Travail et Mobilités) - CNRS.

Altamirano, Carlos (dir.) (2002) Términos críticos de sociología de la cultura, Buenos Aires, Paidós, 269 p.

Bañón Hernández, Antonio Miguel (2002) Discurso e inmigración. Propuestas para el análisis de un debate social, Murcia, Universidad de Murcia, $350 \mathrm{p}$.

Barré, Virginie (2000) Dites-le avec des femmes: le sexisme ordinaire dans les médias, Paris, CFD ; AFJ, $140 \mathrm{p}$.

Battegay, Alain ; Boubeker, Ahmed (1993) Les images publiques de l'immigration : médias, actualité, immigration dans la France des années 80, Paris, L'Harmattan, 192 p.

Bauböck, Rainer ; Rundell, John (ed.) (1998) Blurred Boundaries : Migration, Ethnicity, Citizenship, Aldershot; Brookfield, Ashgate, $356 \mathrm{p}$.

Bernárdez Rodal, Asunción (ed.) (2007) Mujeres inmigrantes en España. Representaciones en la información y percepción social, Madrid, Editorial Fragua, 147 p. (Biblioteca de Ciencias de la Comunicación)

Biagi, Shirley ; Kern-Foxworth, Marilyn (ed.) (1997) Facing Difference : Race, Gender, and Mass Media, Thousand Oaks, Pine Forge Press, 285 p.

Blandin, Claire ; Méadel, Cécile (dir.) (2009), Dossier: La cause des femmes, Le temps des médias, nº12, pp. 6-186.
Bonnafous, Simone (1991) L'immigration prise aux mots. Les Immigrés dans la presse au tournant des années 80, Paris, Kimé, 301 p. (Argumentation et sens du Langage).

Bosshart Louis (1991) Femmes et médias, Fribourg, Université de Fribourg, 194 p. (Communication sociales, Cahiers de travaux pratiques ; 24).

Broussard, Jinx Coleman (2004) Giving a Voice to the Voiceless : Four Pioneering Black Women Journalists, London, Routledge, 215 p.

Byerly, Carolyn ; Ross, Karen (2006) Women and the media. A critical introduction - USA, UK, Malden, Blackwell Publishing, 293 p.

Calvo, Faviola (2001) Apuntes para un análisis de prensa, in Bonelli, E. ; Ulloa M. (ed.) Tráfico e inmigración de mujeres en España. Colombianas y ecuatorianas en los servicios domésticos y sexuales, Madrid, ACSUR-Las Segovias, pp. 51-64.

Carter, Cynthia ; Branston, Gill ; Allan, Stuart (eds.) (1998) News, gender and power, London ; New York, Routledge, 297 p.

Castles, Stephen ; Davidson, Alastair (2000) Citizenship and Migration. Globalization and the Politics of Belonging, London ;bNew York, Routledhe, 258 p.

Chambers, Deborah; Steiner, Linda ; Fleming, Carole (2004) Women and journalism, London, New York, Routledge, 278 p.

Coleman, Stephen ; Normann, Emilie (2000) New Media and Social Inclusion, London, Hansard Society for Parliamentary Government, 43 p.

Cossée, Claire (2010) Médias tsiganes en France et en Hongrie : re-présentation de soi dans l'espace public, Revue européenne des migrations internationales, vol. $26, \mathrm{n}^{\circ} 1$, pp. 5780.

Cossée, Claire (2012a) Les minorités : minoritaires et minorisées. Mise en perspective, Colloque Minorités, Exclusion, Intégration ( $1^{\text {er }}$ juin 2012), Paris, Observatoire des minorités; Université Paris 3 Sorbonne Nouvelle. 
Cossée, Claire (2012b) Introduction. L'expérience minoritaire: le double écueil ethnocentrisme/ androcentrisme, in Cossée, C. ; Miranda, A. ; Ouali, N. ; Séhili, D. (eds.) Le genre au cour des migrations, Paris, Pétra, pp. 247-253 (intersectionS).

Cottle, Simon (2000) Ethnic Minorities and the Media. Changing Cultural Boundaries, Philadelphia ; Maidenhead, Open University Press, $251 \mathrm{p}$.

Damian-Gaillard, Béatrice ; Frisque, Cégolène; Saitta, Eugénie (dir.) (2010) Le journalisme au féminin. Assignations, inventions, stratégies, Rennes, Presses Universitaires de Rennes, $282 \mathrm{p}$.

De Bruin, Marjan ; Ross, Karen (eds.) (2004) Gender and the newsroom cultures. Identities at work, Cresskill, Hampton Press, 276 p.

Dente Ross, Susan; Cuiller, David (2004) Gambling with Identity: American Indian Self-Representations on Tribal Web Sites, in Lind, R. A. (dir.) Race /Gender/Media: Considering Diversity Across Audience, Content, and Producers, Boston, Allyn \& Bacon, pp. $150-157$.

Dijk, Teun Adrianus van (2003) Dominación étnica y racismo discursivo en España y América Latina, Barcelona, Gedisa, 205 p. (Colección Libertad y Cambio)

Djerf-Pierr Monika (2007) The gender of journalism. The structure of logic of the field in the twentieth century, Nordicom Review, pp. 81-104.

Djerf-Pierre Monika (2005) Lonely at the top. Gendered media elites in Sweden, Journalism, vol. 6, n³, pp. 265-290.

Frachon, Claire; Sassoon, Virginie (eds.) (2008) Médias et diversité. De la visibilité aux contenus. Etat des lieux en France, au RoyaumeUni, en Allemagne et aux Etats-Unis, Paris, Karthala ; Institut Panos, 157 p.

Gautier, Gisèle (2007) Rapport d'activité 20062007 : quelle place pour les femmes dans les médias?, Paris, Délégation aux droits des femmes et à l'égalité des chances entre hommes et femmes au Sénat, 174 p.
Giner, Salvador; Lamo de Espinosa, Emilio ; Torres, Cristóbal (2004) Diccionario de Sociología, Madrid, Alianza Editorial Ciencias Sociales, 1030 p.

Guénif Souilamas, Nacira (2007) L'iconographie des Marianne «multicolores». Une arme de destruction massive au service de l'universalisme abstrait, in Rigoni, I. (ed.) Qui a peur de la télévision en couleurs? La diversité culturelle dans les médias, Montreuil, Aux Lieux D'Être, pp. 85-107.

Guénif Souilamas, Nacira; Macé, Eric (2004) Les féministes et le garcon arabe, La Tour d'Aigues, Édition de l'Aube, 106 p. (Monde en cours).

Guillaumin, Colette (1972) L'idéologie raciste, genèse et langage actuel, Paris ; La Haye, Mouton, $247 \mathrm{p}$.

Herne, Claude (1993) La définition sociale de la femme à travers la publicité, Paris, L'Harmattan, 214 p. (Contradictions ; $\mathrm{n}^{\circ} 71-72$ ).

Husband, Charles (1994) A Richer Vision: The Development of Ethnic Minority Media in Western Democracies, Paris, UNESCO, 149 p.

Institut Français de Presse (IFP) (1992) Les journalistes francais en 1990. Radiographie d'une profession, Paris, La Documentation française, $140 \mathrm{p}$.

Institut Français de Presse (IFP) (2001) Les journalistes français à l'aube de l'an 2000 : profils et parcours, Paris, Panthéon Assas, 169 p.

Isin, Engin F. Wood, Patricia K. (1999) Citizenship and Identity, London, Sage Publications, 208 p.

Joppke, Christian (ed.) (1998) Challenge to the Nation-State: Immigration in Western Europe and the United States, Oxford, Oxford University Press, $360 \mathrm{p}$.

Juteau, Danielle (1981) Visions partielles, visions partiales : visions des minoritaires en sociologie ?, Sociologie et sociétés, vol. XIII, $\mathrm{n}^{\circ} 2$, pp. 33-47.

Juteau, Danielle (1999) L'ethnicité et ses frontières, Montréal, Presses Universitaires de Montréal, 226 p. (Trajectoires sociales). 
Lind, Rebecca Ann (2004) Race / Gender / Media: Considering Diversity Across Audience, Content, and Producers, Boston, Allyn \& Bacon, 384 p.

Lavoisier, Bénédicte (1978) Mon corps, ton corps, leur corps: le corps de la femme dans la publicité, Paris, Seghers, 255 p.

Löfgren-Nilsson, Monika (2010) Le genre en pensées et en actes : le cas des informations télévisées suédoises, in Béatrice Damian, Cégolène Frisque, Eugénie Saitta (eds.), Le journalisme au féminin: assignations, inventions et stratégies, Rennes, Presses Universitaires de Rennes, pp. 119-152.

Marchetti, Dominique ; Ruellan, Denis (eds.) (2001) Devenir journalistes: sociologie de l'entrée sur le marché du travail, Paris, La Documentation française, $169 \mathrm{p}$.

Martín Muñoz, Gema (2005) Mujeres musulmanas: entre el mito y la realidad, in Checa Olmos, F. (ed.) Mujeres en el camino: el fenómeno de la migración femenina en España, Barcelona, Icaria, pp. 193-220.

Masanet Ripoll, Erika; Ripoll Arcacia, Carolina (2008) La representación de la mujer inmigrante en la prensa nacional, Papers, no89, pp. 169-185.

Mattelart, Michele (1986) Women, media, crisis, feminity, and disorder, London, Methuen, 120 p. (Comedia Series).

Mattelart, Michele (2003) Femmes et médias: retour sur une problématique, Réseaux, vol. 21, n¹20-4, pp. 23-51.

Morley, David (2000) Home Territories : Media, Mobility and Identity, London, Routledge, $340 \mathrm{p}$.

Navarro, Laura (2008) Los medios de comunicación nacidos de las nuevas migraciones en España, III Anuario de la Comunicación del Inmigrante 08/09, Etnia Comunicación, Madrid, pp. 110-115.

Navarro, Laura (2010) Islamophobia and Sexism: Muslim Women in the Western Mass Media, Human Architecture: Journal of the Sociology of Self-Knowledge, vol. VIII, $\mathrm{n}^{\circ} 2$, pp. 95-114.
Neveu, Erik (2000) Le genre du journalisme. Des ambivalences de la féminisation d'une profession, Politix, vol. 13, n51, pp. 179212.

Oprea, Alexandra (2012) Pour un réexamen de la justice sociale depuis la base: l'expérience des femmes roms, in Cossée, C. ; Miranda, A. ; Ouali, N. ; Séhili, D. (eds.) Le genre au cour des migrations, Paris, Pétra, pp. 293-313 (intersectionS).

Ouali, Nouria; Messian, Annick (1999) Images des femmes immigrées dans les médias francophones, Nouvelle Tribune, $\mathrm{n}^{\circ} 20$ 21, pp. 98-105.

Parlement européen (2008) Résolution du 25 septembre 2008 sur les médias associatifs en Europe 2008/2011, (INI), Bruxelles, P6_TA (2008) 0456.

Pérez, Clara (2003) Las inmigrantes en la prensa: víctimas sin proyecto migratorio, Mugak, $\mathrm{n}^{\circ} 24$ [en ligne] URL: < http://www.pensamientocritico.org/claper 1103.htm>

Rabaud, Aude (2012) Minorités (situation de), in Laacher, Smain (ed.) Dictionnaire de l'immigration en France, Paris, Larousse, pp. 300-315.

Reiser, Michèle; Grésy, Brigitte (2008) Rapport sur l'image des femmes dans les médias, Paris, Commission de réflexion sur l'image des femmes dans les médias, 25 septembre [en ligne] URL: $<$ http://www.ladocumentationfrancaise.fr/ var/storage/rapportspublics/084000614/0000.pdf>

Riggins, Stephen Harold (ed.) (1992) Ethnic Minority Media. An International Perspective, London, Sage Publications, 296 p.

Rigoni, Isabelle (2011) Intersectionality and Mediated Cultural Productions in the Globalised Post-Colonial World, Race and Ethnicity Journal, vol. 35, n5, pp. 834-849 [en ligne, réf. du 14 novembre 2011] URL: $<$ http://www.tandfonline.com/doi/abs/10. $\underline{1080 / 01419870.2011 .628035>}$ 
Rigoni, Isabelle (ed.) (2010a) Migrants, minorités ethniques et Internet. Usages et représentations (Dossier), Migrations Société, vol. 22, n¹32, pp. 33-171.

Rigoni, Isabelle (2010b) Journalisme, militantisme et enjeux des mobilisations solidaires. Genre, religion et classe dans la presse écrite musulmane outre-Manche, in Damian, B. ; Frisque, C. ; Saitta, E. (eds.) Le journalisme au féminin: assignations, inventions et stratégies, Rennes, Presses Universitaires de Rennes, pp. 219-235.

Rigoni, Isabelle (2010c) Les luttes de dénomination autour des "médias des minorités ethniques »: réflexions méthodologiques et épistémologiques, Migrations Société, vol. 22, no 128, pp. 95-110.

Rigoni, Isabelle (ed.) (2007) Qui a peur de la télévision en couleurs? La diversité culturelle dans les médias, Montreuil, Aux Lieux d'Être, 332 p. (Mondes Contemporains).

Rigoni, Isabelle; Berthomière, William; Hily, Marie-Antoinette (eds.) (2010) Les médias des minorités ethniques. Représenter l'identité collective sur la scène publique, Revue européenne des migrations internationales, vol. 26, n¹, pp. 7-139.

Rigoni, Isabelle; Saitta, Eugénie (eds.) (2012) Mediating cultural diversity in a globalised public space, Basingstoke, Palgrave Macmillan, $192 \mathrm{p}$.

Robinson, Gertrude (2004) Gender in the newsroom. The Canadian experiences, in De Bruin M., Ross K. (eds.) Gender and the newsroom cultures. Identities at work, Cresskill, Hampton press, pp. 181-196.
Rodríguez García, Estela (2005) Mujeres inmigradas y medios de comunicación. Movimientos sociales en búsqueda de una representación propia, in Checa Olmos, F. (ed.) Mujeres en el camino: el fenómeno de la migración femenina en España, Barcelona, Icaria, pp. 169-192.

Rodriguez, Clemencia (2001) Fissures in the Mediascape: An International Study of Citizens' Media, Cresskill, Hampton Press, 224 p.

Simon, Pierre-Jean (2006) Pour une sociologie des relations interethniques et des minorités, Rennes, Presses Universitaires de Rennes, 347 p.

Soysal, Yasemin (1994) Limits of Citizenship. Migrants and Postnational Membership in Europe, Chicago, University of Chicago Press, 244 p.

Thiéblemont-Dollet, Sylvie (2009) Le rôle des médias dans l'émergence et la popularisation $\mathrm{du}$ mouvement d'émancipation des femmes immigrées ou d'origine immigrée: l'exemple du mouvement Ni Putes Ni Soumises (20012007), Le temps des médias, n¹2, pp. 141-151. 